\title{
Performance of a Chirped Bragg Grating Based First Order PMD Compensator in WDM Optical Transmission System
}

\author{
${ }^{1}$ M.S. Islam and ${ }^{2}$ S.P. Majumder \\ ${ }^{1}$ Institute of Information and Communication Technology \\ ${ }^{2}$ Department of Electrical and Electronic Engineering, \\ Bangladesh University of Engineering and Technology, Dhaka-1000, Bangladesh
}

\begin{abstract}
In this study, the performance of an adjustable first order polarization mode dispersion compensator has analyticaly evaluated based on a $2 \mathrm{~cm}$ long high birefringence linearly chirped Bragg grating and simulated a 4-channel Wavelength Division Multiplexing (WDM) system using this compensator. The device can adjust Differential Group Delay (DGD) in a linearly continuous way without affecting wavelength outside the bandwidth. The various properties of the device such as relative group delay, reflectivity, DGD are investigated in terms of wavelength and stretching ratio. Results show that under stretched condition; the device generates a time delay between fast and slow polarization axes that is adjustable from $0-55 \mathrm{ps}$ and is tunable within $2.4 \mathrm{~nm}$ wavelength range. Through simulation, it is also found that the power penalty is reduced from $7.10-4.5 \mathrm{~dB}$ at a mean DGD $40 \mathrm{ps}$ for a 4-channel $10 \mathrm{~Gb} \mathrm{sec}^{-1} \mathrm{WDM}$ system when no stretched is applied on the grating.
\end{abstract}

Key words: Differential group delay, polarization mode dispersion, dispersion compensator, fiber Bragg grating, simulation, device

\section{INTRODUCTION}

Polarization Mode Dispersion (PMD) has become one of the most difficult issues in implementing next generation high-bit rate $10 \mathrm{~Gb} \mathrm{sec}{ }^{-1}$ and beyond transmission systems. PMD is a serious problem because a large amount of the installed fibers exhibit PMD values that are several times that of current state of art fibers. Degrading effects that tended to cause catastrophic events even at lower bit rates have become critical concerns of high performance networks. First order PMD (FOPMD) is the time delay between the fast and slow polarization axes and expressed as Differential Group Delay (DGD).

There have been several experiments and/or simulation work to demonstrate FOPMD compensation (Hakki, 1997; Heismann et al., 1998; Wang et al., 2003; Yan et al., 2006). Conventionally to compensate PMD in a WDM system, channels have to be demultiplexed and compensated individually resulting in increased cost and complexity.

Chirped Fiber Bragg Gratings (1999) are useful for PMD and chromatic dispersion compensation without demultiplexing the individual channel. Pan et al. (2002) experimentally demonstrated chirp-free tunable PMD compensation for a $10 \mathrm{~Gb} \mathrm{sec}^{-1}$ signal by using an adjustable Highly-Birefringence (Hi-Bi) nonlinearly chirped FBG in a novel dual-pass configuration that significantly reduces the induced chirp of the FBG. It is shown that a $45 \mathrm{~km}$ link interacting with the FBG induced chirp is reduced from 4.0-0.5 dB. Kun et al. (2002) through numerical simulation assessed the efficiency of a sampled Bragg grating PMD compensator for $10 \mathrm{~Gb} \mathrm{sec}^{-1} \mathrm{NRZ}$ transmission system with 58.6 psand 106 ps DGD, respectively by applying transverse and uniform distributed force.

In this study, analytical formulations has developed and evaluate the performance of a Hi-Bi Linear Chirped Bragg Grating (LCBG) based WDM-FOPMD compensation device and simulation is carried out to investigate the feasibility to compensate several channels at the same time for a WDM transmission system using this device.

The reflection point inside the grating can be moved several millimeters by stretching the grating few microns only. It is found that a $2 \mathrm{~cm}$ long LCBG allows the FOPMD to be adjusted from $0-55 \mathrm{ps}$ in a continuous way while stretching it by $0.2 \%$. Simulation results show that the power penalty for a 4-channel $10 \mathrm{~Gb} \mathrm{sec}^{-1}$ system with $40 \mathrm{ps}$ average DGD is reduced significantly.

Corresponding Author: M.S. Islam, Institute of Information and Communication Technology, Bangladesh University of Engineering and Technology, Dhaka-1000, Bangladesh 


\section{MATERIALS AND METHODS}

WDM-PMD compensation system model: The system model of a LCFBG based PMD compensator is shown in Fig. 1. The proposed delay element consists of a 4-port Polarization Beam Splitter (PBS) and a $2 \mathrm{~cm}$ length Hi-Bi LCFBG. The PBS splits the incoming optical signal into two orthogonal polarizations. The fast axis polarization signal $\left(\mathrm{P}_{\mathrm{f}}\right)$ enters the Hi-Bi LCFBG from the longer wavelength port and slow one $\left(\mathrm{P}_{\mathrm{s}}\right)$ from the shorter wavelength port.

The polarization states of the signal $\left(\lambda_{i}\right)$ within the bandwidth of the grating will be reflected and differently delayed by the grating. By properly tuning the $\mathrm{LCFBG}$, we can adaptively generate required DGD $(\Delta \tau)$ for the PMD compensation. The two orthogonally polarized optical signals are then combined without interference and the compensated signal is directed to output port 1 of the PBS. An optical circulator can be used to separate the input signal and the PMD compensated output signal. All light $\left(\lambda_{0}\right)$ outside the reflection bandwidth of the grating will not be affected and detected to output port 2 . Figure 2 shows the typical module of the compensation scheme using the LCBG based compensator. It shows the power penalty (at the input and output) for different DGD values when the input power is evenly divided between the two PSPs. The power penalty for small (such as $10 \mathrm{ps)}$ DGD is usually negligible or within the power margin of the system and therefore be tolerated. It is the high values of DGD that cause significant degradation when signal power splits almost equally between the two PSPs.

At the input 4-channels (bit rate, 10 Gbps per channel) with wavelength $\lambda_{1}-\lambda_{4}$ are multiplexed and fed to fiber link. In the simulation, we take two span of $200 \mathrm{~km}$ length fiber link. The LCFBG compensators are placed after $200 \mathrm{~km}$ fiber link length where fiber loss is totally compensated by EDFA. Finally, the channels are demultiplexed at the receiving end and eye diagram is monitored without and with compensator to assess the deterioration and amount of compensation of each channel.

\section{Theoretical analysis}

Characteristics of Hi-Bi LCFBG: Let assume that a Bragg grating in a single mode fiber consists of a refractive index $n$ that is varied with the period $\Lambda$ and the modulation amplitude is added to the initial refractive

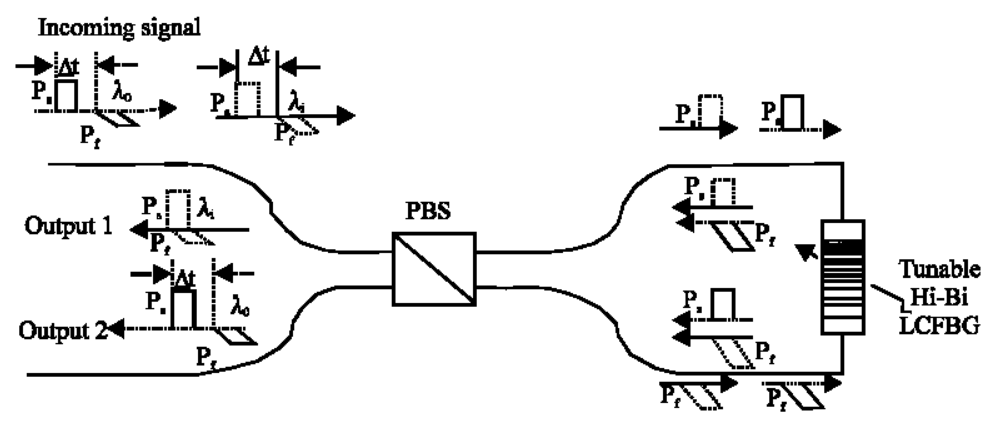

Fig. 1: System model of the LCFBG based PMD compensator. The incoming signal has polarization components along both the fast $\left(\mathrm{P}_{f}\right)$ and slow $\left(\mathrm{P}_{s}\right)$ axis. The LCFBG generates the required DGD for the Bragg reflected signal $\left(\lambda_{i}\right)$ while it does not affect the signal $\left(\lambda_{0}\right)$ outside the grating bandwidth

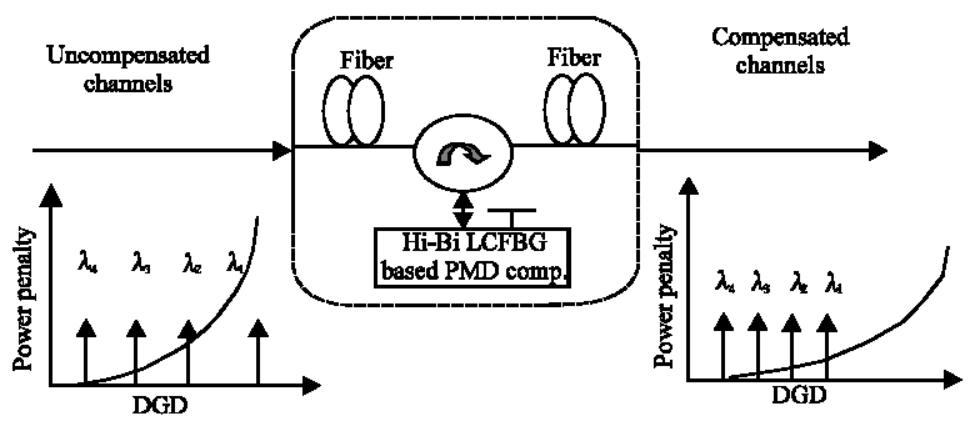

Fig. 2: Hi-Bi LCFBG based WDM-PMD compensation module 
index in the fiber. The perturbation to the effective refractive index $n_{\text {eff }}$ of the guided mode(s) of interest given by:

$$
\delta \mathrm{n}(\mathrm{z})=\delta \mathrm{n}_{\mathrm{eff}}[1+v(\mathrm{z}) \cos (\mathrm{Kz}+\phi(\mathrm{z}))]
$$

Where:

$$
\delta \mathrm{n}_{\mathrm{eff}}=\mathrm{n}_{\mathrm{av}}-\mathrm{n}_{\mathrm{co}}=\text { The average index change over one }
$$
period

$\mathrm{v}=$ The fringe visibility of the index change, $0 \leq \mathrm{v} \leq 1$

$\cos (\mathrm{Kz}=\phi(\mathrm{z}))=$ The index perturbation which has a constant spatial frequency with an additional position dependent phase variation $\varphi(z)$ that represents the change in periodicity

Birefringence in optical fibers is defined as the difference in refractive index $\Delta \mathrm{n}$ between a pair of orthogonal modes (called slow and fast modes) and results from the presence of circular asymmetries in the fiber section.

The refractive index for both the slow and fast-mode is defined as:

$$
\mathrm{n}_{\text {eff, } \mathrm{s}}=\mathrm{n}_{\text {eff }}+\frac{\Delta \mathrm{n}}{2}, \quad \mathrm{n}_{\text {eff }, \mathrm{f}}=\mathrm{n}_{\text {eff }}-\frac{\Delta \mathrm{n}}{2}
$$

where, $\mathrm{n}_{\text {eff }}$ is the fiber effective index. To denote the refractive index of both modes from now on notation $\mathrm{n}_{\text {eff } \mathrm{f}(\mathrm{s})}$, will use.

The effect of strain on Hi-Bi LCFBG: The penetration depth in reflection and the distance traversed during transmission in response to an applied axial strain or temperature changes because there is a redistribution of the period as well a change in the refractive index due to photo-elastic or photo-thermal effect (Kersey et al., 1997). Both of these effects influence the Bragg condition (i.e., $\lambda_{\mathrm{B}}=2 \mathrm{n}_{\text {eff, f(s) }} \Lambda$ ).

Suppose at constant temperature under the influence of axial strain (+ve) $\varepsilon(\mathrm{z})$ at the grating position $\mathrm{z}$, the FBG will experience a physical elongation of grating period, $\Lambda$ and a change of refractive index, $\mathrm{n}_{\mathrm{eff}} \mathrm{f(s)}$ due to the photo-elastic effect. The grating period can be written as:

$$
\Lambda(\mathrm{z}, \varepsilon(\mathrm{z}))=\left(\Lambda_{0}+\mathrm{C}_{0} \mathrm{z}\right)(1+\varepsilon(\mathrm{z}))
$$

where, $\Lambda_{0}$ is the grating period at position, $\mathrm{z}=0$ without strain. Constant $\mathrm{C}_{0}$ denotes the initial chirp of the grating and represented as $\mathrm{C}_{0}=\mathrm{d} \Lambda /\left.\mathrm{dz}\right|_{z=0}$. The induced change in the fiber index $\operatorname{dn}_{\text {eff } \mathrm{f}(\mathrm{s})}(\mathrm{z})$ that is due to the photo-elastic effect is expressed as:

$$
\frac{\mathrm{dn}_{\text {eff, } \mathrm{f}(\mathrm{s})}}{\mathrm{n}_{\mathrm{eff}, \mathrm{f}(\mathrm{s})}}=-\rho_{\mathrm{e}} \varepsilon(\mathrm{z})
$$

where it assumed the photo-elastic contributions into $\rho_{\mathrm{e}}$ which is defined by:

$$
\rho_{\mathrm{e}}=\frac{\mathrm{n}_{\mathrm{eff}, \mathrm{f}(\mathrm{s})}}{2}\left[\mathrm{p}_{12}-\mu\left(\mathrm{p}_{11}+\mathrm{p}_{12}\right)\right]
$$

in terms of Pockel's coefficients $\mathrm{p}_{\mathrm{ij}}$ and $\mu$ is the Poisson ratio. Together with the Bragg condition the resonance condition can be approximated and becomes dependent on local strain. Thus Bragg wavelength $\lambda_{\mathrm{f}(\mathrm{s})}$ at grating position $\mathrm{z}$ becomes:

$$
\lambda_{\mathrm{f}(\mathrm{s})}(\mathrm{z})=2 \mathrm{n}_{\mathrm{eff}, \mathrm{f}(\mathrm{s})}\left[\left(\Lambda_{0}+\mathrm{C}_{0} \mathrm{z}\right)+\left(\Lambda_{0}+\mathrm{C}_{0} \mathrm{z}\right)\left(1-\rho_{\mathrm{e}}\right) \varepsilon(\mathrm{z})\right](4)
$$

From Eq. 4 it is straightforward to observe that the reflection wavelength shift of the grating is proportional to the applied strain.

If strain gradient is added to a FBG, the Bragg wavelength varies linearly along the fiber length because of the change in the effective grating period.

Modeling of the fiber bragg grating: Coupled-mode theory is a good tool for obtaining quantitative information about the diffraction efficiency and spectral dependence of fiber grating.

Generally, Bragg reflection is the interaction between the optical signal and its medium. The wave formulation for forward and backward wave amplitudes $F(z)$ and $B(z)$ can be expressed as:

$$
\begin{gathered}
\frac{d F^{+}(z)}{d z}=-i \hat{\sigma}_{f(s)} F^{+}(z)+i \kappa_{f(s)}(z) B^{+}(z) \\
\frac{d B^{+}(z)}{d z}=-i \hat{\sigma}_{f(s)} B^{+}(z)-i \kappa_{f(s)}^{*}(z) F^{+}(z) \\
F^{+}(z)=F(z) e^{(i \delta z-\varphi / 2)}, B^{+}(z)=B(z) e^{(-i \delta z+\varphi / 2)}
\end{gathered}
$$

Where:

$\mathrm{F}^{+}(\mathrm{z}) \quad=$ The (reference) represents the forward propagating mode

$\mathrm{B}^{+}(\mathrm{z})=$ The (signal) is the identical backward (counter) propagating mode

$\hat{\sigma}_{\mathrm{f}(s)}$ andK $\mathrm{K}_{\mathrm{f}(s)}=$ The general dc (period averaged) self coupling and ac coupling coefficients, respectively. It can be expressed as: 


$$
\begin{array}{r}
\hat{\sigma}_{\mathrm{f}(\mathrm{s})}=\delta_{\mathrm{f}(\mathrm{s})}+\sigma_{\mathrm{f}(\mathrm{s})}-\frac{1}{2} \frac{\mathrm{d} \varphi(\mathrm{z})}{\mathrm{dz}} \\
=\delta_{\mathrm{f}(\mathrm{s})}+\frac{2 \pi}{\lambda_{\mathrm{f}(\mathrm{s})}} \delta \mathrm{n}_{\mathrm{eff}, \mathrm{f}(\mathrm{s})}-\frac{1}{2} \frac{\mathrm{d} \phi(\mathrm{z})}{\mathrm{dz}} \\
\kappa_{\mathrm{f}(\mathrm{s})}=\frac{\pi}{\lambda_{\mathrm{f}(\mathrm{s})}} \delta \mathrm{n}_{\mathrm{eff}, \mathrm{f}(\mathrm{s})} v \\
\delta_{\mathrm{f}(\mathrm{s})}=\beta-\beta_{\mathrm{B}}=2 \pi \mathrm{n}_{\text {eff, } \mathrm{f}(\mathrm{s})}\left(\frac{1}{\lambda_{\mathrm{f}(\mathrm{s})}}-\frac{1}{\lambda_{\mathrm{B}}}\right)
\end{array}
$$

Where:

$\delta_{\mathrm{f}(\mathrm{s})}=$ The detuning (which is independent of $\mathrm{z}$ for all gratings) from the Bragg wavelength $\lambda_{\mathrm{B}}$ related to period $\Lambda_{0}$

$\delta n_{\text {eff } f(s)}=$ The dc index spatially averaged over a grating period

$\sigma_{\mathrm{f}(\mathrm{s})} \quad=$ The dc coupling coefficient and the derivative $1 / 2(d \varphi(z) / d z$ describes the possible chirp of the grating

The coupled mode equations for the forward and the backward propagating modes can be solved using appropriate boundary conditions.

The boundary conditions assume a forward propagating mode with $\mathrm{F}^{+}(0)=1$ and that the backward propagating mode at the end of the grating, will be zero, $\mathrm{B}^{+}\left(\mathrm{L}_{\mathrm{g}}\right)=0$ as there are no perturbing beyond the end of the grating. The solution of the coupled mode Eq. 5 can be best expressed by:

$$
\left[\begin{array}{c}
\mathrm{F}^{+}(0)=1 \\
\mathrm{~B}^{+}(0)
\end{array}\right]=[\mathrm{T}]\left[\begin{array}{c}
\mathrm{F}^{+}\left(\mathrm{L}_{\mathrm{g}}\right) \\
\mathrm{B}^{+}\left(\mathrm{L}_{\mathrm{g}}\right)=0
\end{array}\right]
$$

where, $\mathrm{T}$ is the transfer matrix and given by:

$$
\mathrm{T}=\left[\begin{array}{ll}
\mathrm{T}_{11} & \mathrm{~T}_{12} \\
\mathrm{~T}_{21} & \mathrm{~T}_{22}
\end{array}\right]
$$

Where:

$$
\begin{gathered}
\mathrm{T}_{11}=\cosh \left(\gamma \mathrm{L}_{\mathrm{g}}\right)-\mathrm{i} \frac{\hat{\sigma}_{\mathrm{f}(\mathrm{s})}}{\gamma} \sinh \left(\gamma \mathrm{L}_{\mathrm{g}}\right) \\
\mathrm{T}_{12}=-\mathrm{i} \frac{\kappa_{\mathrm{f}(\mathrm{s})}}{\gamma} \sinh \left(\gamma \mathrm{L}_{\mathrm{g}}\right) \\
\mathrm{T}_{22}=\cosh \left(\gamma \mathrm{L}_{\mathrm{g}}\right)+\mathrm{i} \frac{\hat{\sigma}_{\mathrm{f}(\mathrm{s})}}{\gamma} \sinh \left(\gamma \mathrm{L}_{\mathrm{g}}\right) \\
\mathrm{T}_{21}=\mathrm{i} \frac{\mathrm{K}_{\mathrm{f}(\mathrm{s})}}{\gamma} \sinh \left(\gamma \mathrm{L}_{\mathrm{g}}\right)
\end{gathered}
$$

Where:

$\gamma=$ The parameter relating to coupling coefficient as:

$$
\gamma=\sqrt{\kappa_{\mathrm{f}(\mathrm{s})}^{2}-\hat{\sigma}_{\mathrm{f}(s)}^{2}}
$$

Reflectivity is the percentage of light reflected at the Bragg wavelength, the wavelength outside of the reflected bandwidth is transmitted without disturbance. For the case of uniform grating, the complex amplitude reflection coefficient at the beginning of the FBG at $z=0$ is defined as:

$$
\begin{gathered}
\rho_{\mathrm{f}(\mathrm{s})}(\lambda)=\frac{\mathrm{B}^{+}\left(\hat{\sigma}_{\mathrm{f}(\mathrm{s})}\right)}{\mathrm{F}^{+}\left(\hat{\sigma}_{\mathrm{f}(\mathrm{s})}\right)}=\frac{\mathrm{T}_{12}}{\mathrm{~T}_{11}} \\
\rho_{\mathrm{f}(\mathrm{s})}(\lambda)=-\frac{\kappa_{\mathrm{f}(\mathrm{s})} \sinh \left(\gamma \mathrm{L}_{\mathrm{g}}\right)}{\hat{\sigma}_{\mathrm{f}(\mathrm{s})} \sinh \left(\gamma \mathrm{L}_{\mathrm{g}}\right)+\mathrm{i} \gamma \cosh \left(\gamma \mathrm{L}_{\mathrm{g}}\right)}
\end{gathered}
$$

The reflectivity or the power reflection coefficient, $\mathrm{R}_{\mathrm{f}(\mathrm{s})}(\lambda)$ is given by:

$$
R_{f(s)}(\lambda)=\left|\rho_{f(s)}(\lambda)\right|^{2}=\frac{\sinh ^{2}\left(\gamma L_{g}\right)}{\cosh ^{2}\left(\gamma L_{g}\right)-\hat{\sigma}_{f(s)}^{2} / \kappa_{f(s)}^{2}}
$$

The group delay of the reflected light can be determined from the phase of the complex amplitude reflection coefficient $\rho_{\mathrm{f}(\mathrm{s})}(\lambda)$. If $\varphi_{\mathrm{p}}=$ phase $\left(\rho_{\mathrm{f}(\mathrm{s})}(\lambda)\right.$ then the time delay $\tau_{R}$ for light reflected off of a grating is:

$$
\tau_{\mathrm{R}, \mathrm{f}(\mathrm{s})}=\frac{\mathrm{d} \varphi_{\rho}}{\mathrm{d} \omega}=-\frac{\lambda_{\mathrm{f}(\mathrm{s})}{ }^{2}}{2 \pi \mathrm{c}} \frac{\mathrm{d} \varphi_{\rho}}{\mathrm{d} \lambda_{\mathrm{f}(\mathrm{s})}}
$$

Where:

$$
\begin{array}{ll}
\tau_{\mathrm{R}, \mathrm{f}(\mathrm{s})} & =\text { Usually given in picoseconds } \\
\omega & =\text { The angular frequency } \\
\mathrm{c} & =\text { The velocity of light }
\end{array}
$$

Thus an optical wave traveling through a medium of length $\mathrm{L}$ and refractive index $\mathrm{n}$ will undergo a phase change $\varphi_{\rho}=\left(2 \pi n_{\text {eff } \mathrm{f}(\mathrm{s})} \mathrm{L}_{\mathrm{g}}\right) / \lambda$; the derivative of the phase with respect to wavelength is an indication of the delay experienced by the wavelength component of the reflected light:

$$
\frac{\mathrm{d} \varphi_{\rho}}{\mathrm{d} \lambda_{\mathrm{f}(\mathrm{s})}}=-\frac{2 \pi \mathrm{n}_{\mathrm{eff}, \mathrm{f}(\mathrm{s})} \mathrm{L}_{\mathrm{g}}}{\lambda_{\mathrm{f}(\mathrm{s})}^{2}}
$$

The reflected spectrum is characterized by a main peak at the wavelength defined as $\lambda_{\max , f_{(s)}}=$ 
$2\left(\mathrm{n}_{\text {eff } \mathrm{f}(\mathrm{s})}+\delta \mathrm{n}_{\text {eff } \mathrm{f(s}}\right) \Lambda(\mathrm{z})$. Thus with a large refractive index difference $\Delta \mathrm{n}$ between the fast and slow polarization axes that results in a shift in Bragg wavelength $\Delta \lambda_{\mathrm{B}}=\mid \lambda_{\max , \mathrm{s}}$ $\lambda_{\max } \mathrm{f}=2 \Delta \mathrm{n} \Lambda_{0}$ at the same location for two polarizations. Therefore, the Hi-Bi linearly chirped FBG can be seen as two different chirped FBGs because of the birefringence. The position difference of the reflection produces a DGD:

$$
\operatorname{DGD}(\lambda)=\left|\tau_{\mathrm{R}, \mathrm{f}}(\lambda)-\tau_{\mathrm{R}, \mathrm{s}}(\lambda)\right|
$$

\section{RESULTS AND DISCUSSION}

Following the analytical formulations, at first the performance of a $2 \mathrm{~cm}$ long LCBG is investigated in terms of relative group delay, DGD and stretching to determine the tuning range and maximum amount of FOPMD that can be compensated. To demonstrate its capability simulation is done using Rsoft's OptiSim simulation software for a 4-channel WDM system using this LCBG compensator at unstretched condition.

Figure 3 shows the relative time delay for the fast and slow axes polarization reflected signals inputting from the long and short wavelength ports of the unstressed FBG respectively as a function of wavelength. From the plots, it is seen that the group delay of the grating reveals good linearity and the delay curves for the two polarization axes are shifted by $0.25 \mathrm{~nm}$ at wavelength $1550.5 \mathrm{~nm}$ relative to each other due to the high birefringence of the fiber. The reflection spectrum of the grating is shown in Fig. 4. It is

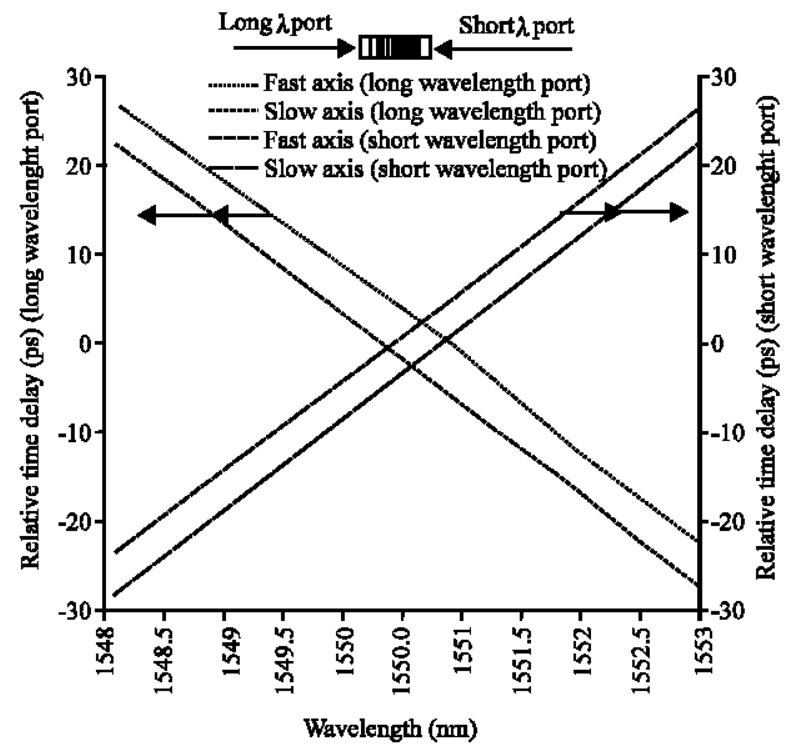

Fig. 3: Reflected relative time delay for the fast and slow axes of polarization observed that the bandwidth of the grating is 1549$1551.02 \mathrm{~nm}$ at unstretched condition. When the grating is stretched, the amplitude and the group delay spectrum shifts to longer wavelength. Note that due to tuning though the pass band shifts to shorter or longer wavelengths but the polarization of the reflected signal remains same.

The change in the FOPMD in terms of DGD as a function of the grating stretching ratio is shown in Fig. 5. By changing the strain $\varepsilon$ from $0-0.2 \%$ the DGD can be continuously adjusted from 3-58 ps. As mentioned earlier, all light outside the reflection bandwidth $\lambda_{0}$ can be directed to the output port without adding any FOPMD. For this system we have a residual DGD of $3 \mathrm{ps}$. It is also found that stretching the LCBG adjust the differential time delay without altering the polarization.

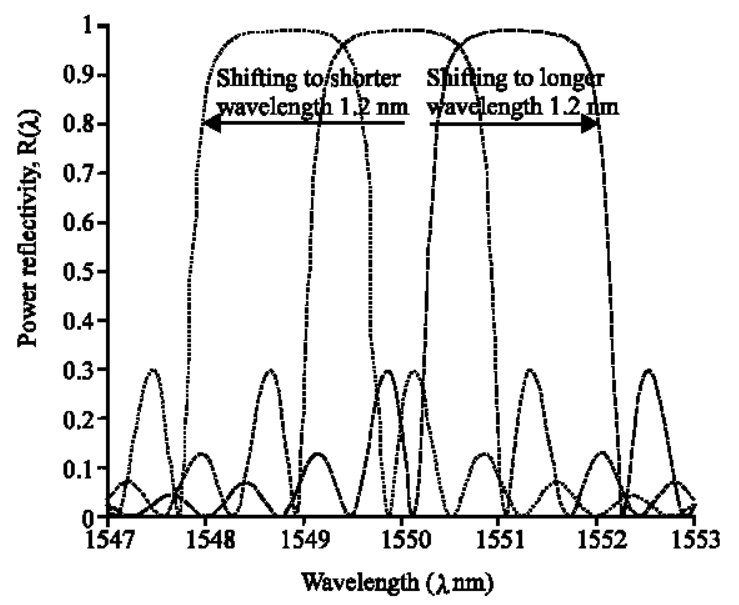

Fig. 4: Reflection spectrum for the LCBG under stretching and compression

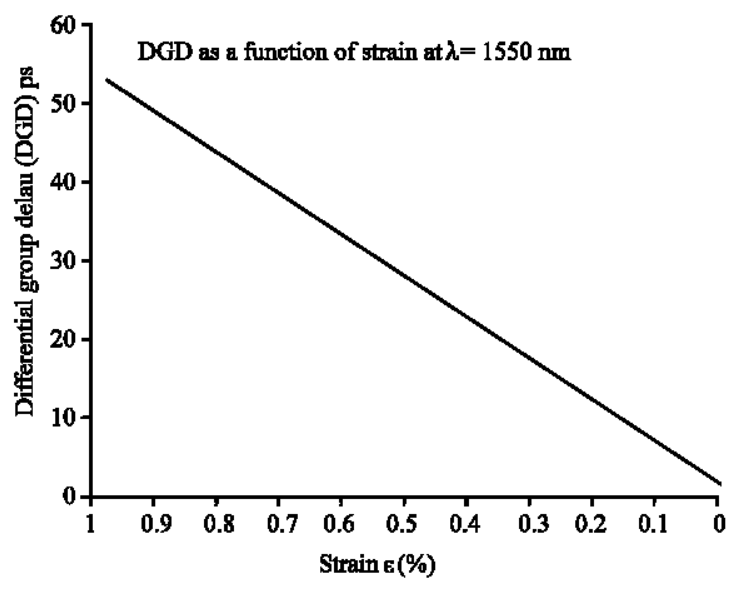

Fig. 5: Variation of the DGD between two polarizations of the LCBG under stretched condition 

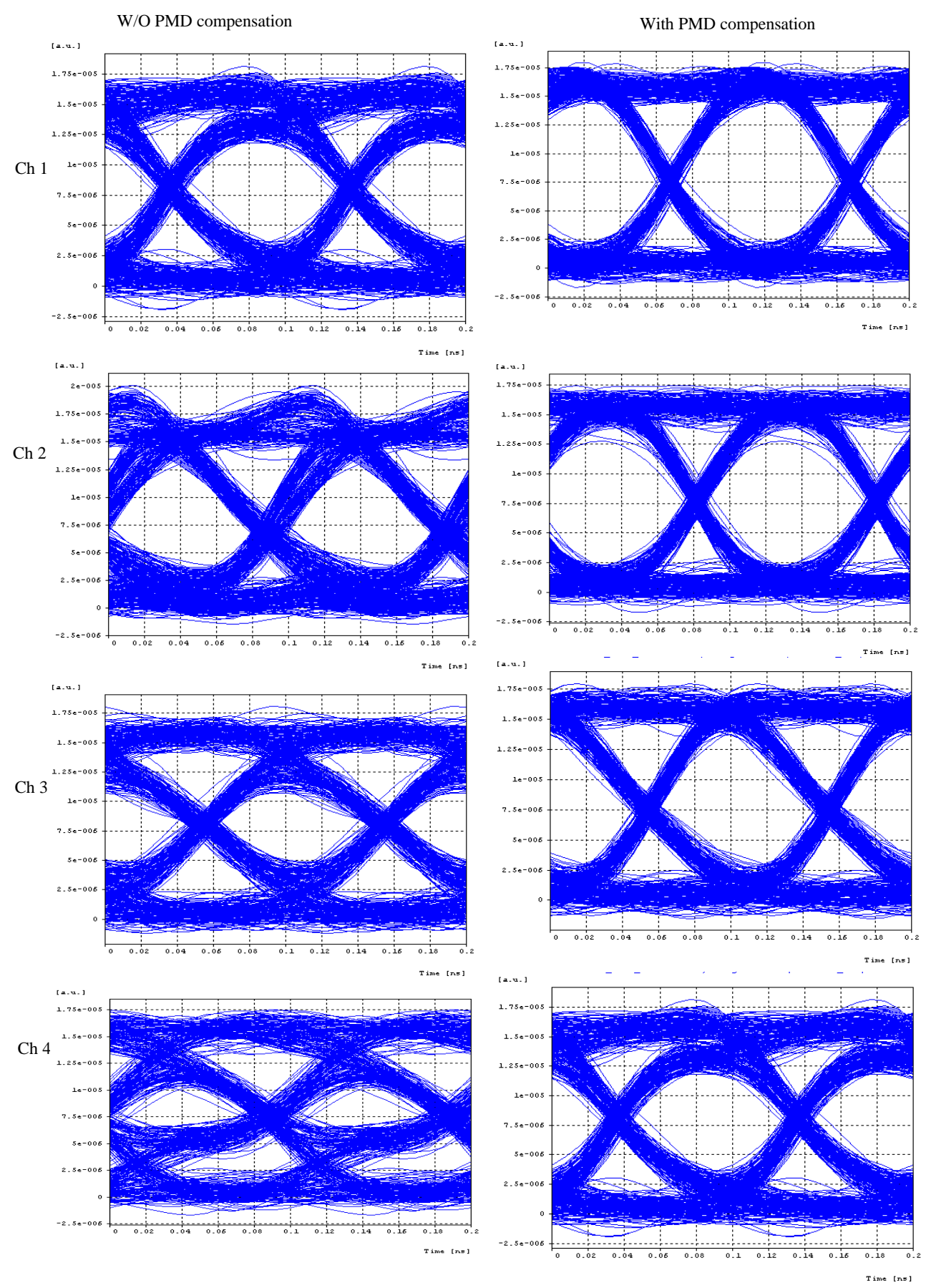

Fig. 6: Eye diagram for a 4-channel (each channel at $10 \mathrm{~Gb} \mathrm{sec}^{-1}, \mathrm{NRZ}$ modulation) with and without WDM-FOPMD compensation

Through simulation, the impact of FOPMD on the WDM system is investigated in terms eye diagram without applying any stress on the LCBG. At the receiving end it demultiplexed all the 4-channel and monitor their eye diagram without and with applying the
FOPMD compensation scheme. A typical measurement of the eye diagram of the accumulated DGD for 4-channels before and after compensation is shown in Fig. 6. At $10 \mathrm{~Gb} \mathrm{sec}^{-1}$ data rate each channel produces $(200 \mathrm{~km}$ span) about $28 \mathrm{ps}$ of DGD and the designed LCBG 


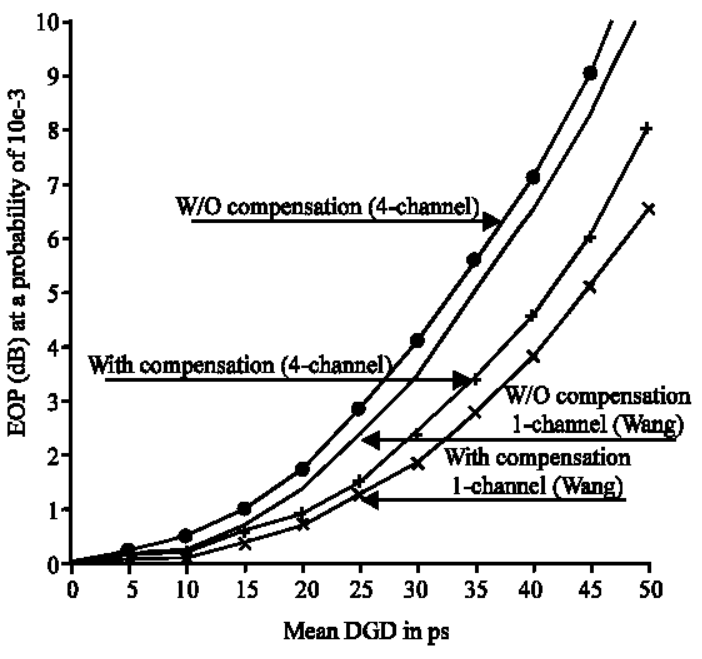

Fig. 7: Eye Opening Penalty (EOP) at a probability of $10^{-3}$ in the dependence of the mean DGD for 4-channel and single transmission system

compensator can compensate only 25 ps DGD without any external strain. It observe that channel 4 has been improved significantly after compensation without impacting the other channels and about 6 ps DGD of each channel remain uncompensated which is also within tolerance level or power margin of the system. Finally, the average Eye-Opening Penalty is calculated (EOP) due to FOPMD for a 4-channel WDM system. Here, the parameter of the Eye-Opening Penalty (EOP) is defined as:

$$
\mathrm{EOP}=-20 \log \left(\frac{\mathrm{B}}{\mathrm{B}_{0}}\right)
$$

where, $\mathrm{B}$ is the eye opening without FOPMD effect and $\mathrm{B}_{0}$ is the eye-opening with FOPMD. Figure 7 shows a comparison of EOP penalty between the 4-channels simulated transmission system and with single channel LCBG based compensation system by Wang et al. (2003) as a function of DGD.

From the curve It is found that about $2.6 \mathrm{~dB}$ (reduced from $7.10-4.5 \mathrm{~dB}$ ) of EOP reduction is obtained at $40 \mathrm{ps}$ by this compensation scheme. The performance of the single channel is $0.55 \mathrm{~dB}$ better for mean DGD $>40$ ps and this happens due to inter-channel crosstalk.

\section{CONCLUSION}

The performance analysis of a novel FOPMD using a $2 \mathrm{~cm}$ long LCBG compensator and 4 channel WDMFOPMD system simulation is carried out. The various properties like- reflectivity, relative group delay and DGD of the Hi-Bi LCBG have been discussed in detail. It is found that the FOPMD compensation capability of the device mainly determined by the characteristics of the Hi-Bi LCBG and improved capability can be achieved by adopting higher chirp ratio phase mask to fabricate the Hi-Bi grating.

The performance of the simulated compensation scheme achieved a significant improvement (i.e., $2.6 \mathrm{~dB}$ ) in the quality of eye pattern of the received signal and it compensates about $50 \mathrm{ps}$ of DGD per channel.

It is also observed that at 40 ps of DGD, the amount eye power penalty for a single channel is about $0.55 \mathrm{~dB}$ less than that of 4-channel WDM system and this happens due to the absence of channel crosstalk in a single channel system. It is an all fiber FOPMD compensation solution which is inexpensive, compact, absence of nonlinear effects and feasible for continuously adjustable DGD.

\section{REFERENCES}

Fiber Bragg Gratings, 1999. Raman Kashyap. 1st Edn., Chirped Fiber Gratings, Academic Press, San Diego, USA., ISBN: 0-12-400560-8, pp: 311-347.

Hakki, B.W., 1997. Polarization mode dispersion compensation by phase diversity detection. IEEE Photonics Technol. Lett., 9: 121-123.

Heismann, F., D.A. Fishman and D.L. Wilson, 1998. Automatic compensation of first-order polarization mode dispersion in a $10 \mathrm{~Gb} / \mathrm{s}$ transmission system. Proc. 24th Eur. Conf. Optical Commun., 1: 529-530.

Kersey, A.D., M.A. Devis and H.J. Patrick, 1997. Fiber grating sensors. J. Lightwave Technol., 15: 1442-1463.

Kun, X., F. Jia, X. Chen, M. Jin, M. Chen, X. Li and S. Xie, 2002. A novel adjustable PMD compensation using sampled Bragg gratings with uniform grating period. Optics Commun., 202: 297-302.

Pan, Z., Y. Xie, S. Lee and A.E. Willner, 2002. Chirp-free tunable PMD compensation using Hi-Bi nonlinearlychirped FBGs in a dual-pass configuration. Optical Fiber Commun. Conf., 3: 113-115.

Wang, M., T. Li and S. Jian, 2003. Tunable PMD compensator based on high-birefringence linearly chirped FBG with cantilever beam. Optics Express, 11: 2354-2362.

Yan, Y., X.S. Yao, M.C. Hauer and A.E. Willner, 2006. Practical solutions to polarization-mode-dispersion emulation and compensation. J. Lightwave Technol., 24: 3992-4005. 